\title{
¿LA MODALIDAD RELEGADA O LA FUNCIÓN PROTOTÍPICA RECALCADA? ACERCA DE LA OPOSICIÓN PRETÉRITO PERFECTO/PRETÉRITO INDEFINIDO/PRETÉRITO IMPERFECTO DE INDICATIVO EN LOS MÉTODOS DE ELE
}

\begin{abstract}
Baran Marek, ¿La modalidad relegada o la función prototipica recalcada? Acerca de la oposición pretérito perfecto/pretérito indefinido/pretérito imperfecto de indicativo en los métodos de ELE [The relegated modality or the prototypical function emphasised? Pretérito perfecto, pretérito indefinido, pretérito imperfecto de indicativo in the ELE teaching]. Studia Romanica Posnaniensia, Adam Mickiewicz University Press, Poznan, vol. XXXI: 2004, pp. 147-154. ISBN 83-232-1353-4, ISSN 0137-2475.
\end{abstract}

The subject of the present article is the place which is attributed to the modality in pedagogical approaches of the system of past tenses in Spanish. This analysis indicates that in ELE handbooks, very often, the comparison between Pretérito Perfecto and Pretérito Indefinido is based on time criteria without taking into consideration the pragmatics, such as: the relation established by the speaker between himself and the statement he makes. Besides, the idea of temporality, suggested there, has little to do with linguistic temporality. Which in turn leads to the falseness on the level of discourse and language categories.

\section{EL INTERCAMBIO COMUNICATIVO, LA PERSPECTIVA FUNCIONAL Y OTROS TÓPICOS DE LA LINGÜÍSTICA APLICADA A LA ENSEÑANZA O LO QUE QUEDA EN EL AIRE}

Lo comunicativo y lo funcional podrían considerarse hoy en día como conceptos y palabras clave de los enfoques dominantes en la enseñanza de lenguas extranjeras. Tanto en los acercamientos teóricos como en los métodos concretos que se proponen para la actividad didáctica, se hace hincapié en la necesidad de desarrollar las cuatro destrezas partiendo del principio según el cual la dimensión comunicativa reforzada por la presentación de las situaciones reales del intercambio lingüístico debe ocupar un lugar privilegiado. En consecuencia, los autores de manuales subrayan que, por ejemplo, los contenidos y estructuras gramaticales se 
presentan junto a los aspectos funcionales y discursivos para satifacer así las necesidades del alumno. Se ha repetido hasta la saciedad que la lengua que se aprende debería servir de instrumento, así que en los índices de los distintos métodos, los objetivos vienen formulados en los términos de los infinitivos referidos a las destrezas por adquirir: saludar, aceptar una invitación, valorar, describir características y actividades en el pasado, podrían servir de ejemplo. "Con la lengua se hacen las cosas", así sería posible resumir el lema de la didáctica moderna, lo que por su parte remite directamente a la pragmática cuyo reflejo se percibe también en otros conceptos recurrentes de la lingüística aplicada a la enseñanza (actos de habla, intenciones comunicativas, contexto...).

\section{LA MODALIDAD O MANOS A LA OBRA}

He decidido centrarme en los tiempos del pasado para comprobar en qué medida los principios que se postulan en los enfoques comunicativos quedan transgredidos en los métodos de Español Lengua Extranjera; los métodos que, desde luego, anuncian la aplicación de dichos principios. Al recurrir al término de transgresión, dejo explícitas las interrogantes que he formulado al respecto; así pues, cabría preguntarse si en varias ocasiones no se va confundiendo la lengua y lo que corresponde al mundo extralingüístico y si realmente la posición y la actitud del hablante se toman en cuenta a la hora de proponer el empleo de uno u otro tiempo del pasado. A mi modo de ver, el cuestionamiento sobre la participación del enunciador queda en una estrecha relación con los fundamentos del acercamiento comunicativo mencionados más arriba: al estudiante se le proporciona un determinado número de elementos léxico-gramaticales para que pueda actuar aprovechando esas herramientas lingüísticas. De esta forma, nos estamos acercando al terreno de la actitud modal y de la modalidad como tal. Al actuar verbalmente, recurrimos a lo que nos ofrece la lengua, utilizando unas u otras unidades del sistema; no hay que olvidar, sin embargo, que somos efectivamente nosotros los que "utilizamos" o "recurrimos" a los elementos sistémicos, dejando una huella indeleble en lo que decimos. Los actos de habla en cuanto procesos de verbalización y categorización se realizan a partir de las unidades de sistema y según ciertas reglas y modelos semántico-sintácticos, pero como señala, entre otros, W. Nowikow (2001: 33) al mismo tiempo en función de las intenciones y creencias de los interlocutores y de acuerdo con las finalidades informativas y coherencia textual.

¿Por qué los tiempos del pasado vinculados al concepto de la modalidad? La premisa previa fue la observación de los valores que se adscriben a los pretéritos en los métodos de ELE, los valores que, a primera vista, parecen mayoritariamente temporales. La perspectiva temporal parece concordar con la visión estereotipada del tiempo gramatical propia del alumnado que suele ver en esa categoría un reflejo más o menos directo del tiempo ontológico ("el tiempo gramatical sitúa los 
acontecimientos en el tiempo como tal"; "el tiempo indica en qué momento sucedió uno u otro evento"). Desde luego, no confiero a esta perspectiva ningún jucio valorativo, el alumno en situación de aprendizaje no tiene que recurrir a todo un fondo conceptual o metalinguístico propio de la reflexión didáctica. La puesta en relieve del elemento temporal podría indicar que en numerosos casos se va perdiendo la dimensión modal de los tiempos del pasado. Esa pérdida no debería corresponder necesariamente a una evidente deficiencia en la exposición de lo que es el sistema temporal del español, dado que en cada método se está efectuando la selección de contenidos con la ascención simultánea de aquellos elementos que se consideran como primarios o básicos para el sistema linguístico en cuestión. De ese modo, podrían formularse tres hipótesis:

1) para la adquisición del sistema de los pretéritos, los métodos de ELE recurren principalmente al concepto de temporalidad, la temporalidad no tanto lingüística como la relacionada con factores extralingüísticos (posteriormente, explicaré con más detalle lo que se entiende por la temporalidad linguística y nolingüística);

2) dicha elección debería considerarse como un punto débil en la exposición del sistema de los tiempos del pasado dado que, en determinados casos, quedan en supenso las intenciones comunicativas del hablante o su postura frente a lo dicho;

3) la minusvaloración de los factores modales resulta de una elección consciente y premeditada debida a la puesta en relieve de las denominadas funciones prototípicas de los pretéritos españoles.

Con tal de encontrar las respuestas satisfactorias a los cuestionamientos así formulados he optado por analizar cinco métodos de ELE (la mayor parte en dos niveles); eran: Avance - Curso de Español, nivel intermedio, Ele 1,2; Español sin fronteras 1,2; Intercambio 1,2; Rápido - Curso intensivo de Español (todos esos métodos van dirigidos a estudiantes adolescentes y adultos, y en sus respectivas presentaciones acentúan la doble consideración de la lengua como sistema y como instrumento de comunicación, lo que por su parte lleva a dar prioridad compartida a los contenidos gramaticales y funcionales). Mi punto de mira ha sido ante todo la oposición que se está estableciendo entre el pretérito perfecto (PP) y el pretérito indefinido (IND), y el empleo del imperfecto de indicativo (IMP) (considerado en abstracción a los demás pretéritos o en oposición al pretérito indefinido, la oposición que sobresale en los manuales de ELE).

\section{LOS TIEMPOS VERBALES Y LOS ACONTECIMIENTOS O LA FALSEDAD DISCRETAMETE SUGERIDA}

La oposición entre el PP y el IND en un número elevado de casos viene determinada en función de marcadores temporales que se sugieren para cada uno de los pretéritos. En los esquemas de los manuales, encontramos un binarismo que podría ilustrarse de la siguiente manera: 
INDEFINIDO, se usa cuando

el hablante sitúa la acción con:

ayer/anteayer/anoche...

el otro día...

el lunes/el martes

el día $17 / 18 \ldots$

la semana pasada

el mes pasado

el verano pasado/...

el año pasado

en $1986 / 87 \ldots$

en agosto/septiembre

hace unos años/tres días...
PRETÉRITO PERFECTO, se usa

cuando el hablante sitúa la acción con:

hoy

a las doce/cuatro...

esta mañana/tarde...

esta semana

este mes

este año

este otoño/verano...

hace un rato/una hora/.;

o se usa si no interesa cuándo ha sucedido

algo:

he estado cuatro veces en Berlín.

¿Has comido paella alguna vez?

La oposición presentada se inspira en la exposición que se hace de ambos tiempos en Intercambio 2, pero la mayoría de los métodos analizados coincide en el principio de marcar la línea divisoria recurriendo a los marcadores temporales, con la contraposición de hoy a ayer. A primera vista, son las circunstancias de tiempo las que configuran el acto comunicativo, de ellos dependería la interpretación de un gran número de enunciados. Los factores extralingüísticos que giran principalmente en torno a los acontecimientos, sucesos o eventos parecen constituir la pista para la elección de uno u otro tiempo verbal. Ese modo de ver la temporalidad no concuerda necesariamente con el concepto de temporalidad lingüística que debería considerarse como categoría gramatical deíctica donde la orientación de una situación se está llevando a cabo respecto a un punto central o el denominado origen (este último no queda establecido de forma absoluta puesto que es a menudo el enunciador mismo el que lo viene determinando). Habrá, entonces, que recordar una afirmación nada novedosa pero, a lo peor descuidada por la didáctica de lenguas extranjeras, de acuerdo con la que los tiempos verbales tienen muy poco que ver con las características de las acciones; los tiempos verbales hablan ante todo del estatuto que quiere atribuir el enunciador a lo que va diciendo. Una de las pocas obras de referencia que no solamente toma en cuenta ese factor sino lo está aplicando en sus exposiciones es Gramática Comunicativa del español de Francisco Matte Bon (1995) en la que, en varias ocasiones, se subraya que una misma acción se puede expresar lingüísticamente con distintos tiempos verbales según el contexto y las intenciones del hablante. El sistema verbal aparece indudablemente como la herramienta del acto comunicativo, pero no hay que olvidar que es efectivamente la herramienta de la que dispone el enunciador que controla y filtra todo lo que dice (F. Matte Bon, 1995: VIII). Es bien sabido que con los marcadores temporales que remiten a unidades de tiempo consideradas como inacabadas, se prefiere emplear el 
PP; podríamos, no obstante, imaginarnos fácilmente la situación cuando el enunciador dentro de la unidad de tiempo considerada como <hoy> dice: Fui a recoger el coche del taller (a lo mejor añadiendo también esta mañana [fui a recoger el coche del taller]) ${ }^{\prime}$. La elección del IND en este caso, se explica desde luego por la intención del hablante de contar el hecho en sí y sin relación con su presente aunque la unidad < día> aún no haya acabado. Evidentemente, si le interesa enumerar todo lo que ha hecho durante el día, p.ej. para que se entienda que está cansado, podrá decir: Esta mañana he ido a recoger el coche del taller. Como sybraya otra vez F. Matte Bon (1995: 114) hay que tomar conciencia que los marcadores temporales como operadores en la elección de los pretéritos constituyen un criterio más bien subjetivo, ya que el enunciador puede, según el momento y el tema, la intención, etc. que concentre su atención, tomar unidades de tiempo más o menos amplias. La oposición PP / IND es algo exquisitamente lingüístico, que se da en el nivel en el que la lengua remite a sí misma y no en su dimensión referencial. F. Matte Bon (1995: 115) lo concluye así:

Si se intenta a toda costa encontrar en el mundo real del que hablamos con la lengua una oposición que en él no existe, se caerá, inevitablemente, en aproximaciones y errores como creer que cl pretérito perfecto se refiere a acciones más recientes. No hay que buscar esta oposición en las acciones y los acontecimientos extralingüisticos en sí, sino en lo que hace el hablante con ellos al evocarlos lingǘsticamente.

Bajo esta óptica, quedan más claros los empleos de tipo: ¡Mamá, me ha 'pegaooo! (grafía original), iQue me han robado el bolso! (Ele 2, 1998: 83) que también por el contexto situacional indican que el enunciador se refiere a cosas pasadas que le interesan por su relación con el presente de la enunciación y que siguen vigentes ${ }^{2}$. Además, el enfoque modal presenta la ventaja de proporcionar una explicación válida y convincente al empleo del PP o del IND sin ningún tipo de marcador temporal. Si estoy buscando a un compañero de trabajo, preguntaré a otro: ¿Has visto a Nacho? (parafraseando: ¿estás en condiciones de ayudarme a encontrar a Nacho, es que tengo que hablar con él); diré, Vi a Nacho, cuando quiera informar sobre ese hecho en sí, sin añadir nada más.

Estas observaciones remiten otra vez al concepto de modalidad cuyo componente pragmático es obvio. La postura del hablante, tanto frente a la

' Aunque no faltan gramáticos que considerarían tal enunciado como agramatical.

${ }^{2}$ En el método mencionado se propone toda una lección que trata efectivamente la oposición entre el PP y el IND (es, precisamente la lección 7). En los empleos situacionales que se proponen, se percibe claramente el vínculo entre el PP y el presente: en las audiciones se interroga a un par de personas por lo qué les ha ocurido, distintas exclamaciones ( $Q$ Qué rabia!, ;Menudo rollo!, iQué día!) junto con las formas verbales señalan que el acontecimiento pasado sigue siendo relevante para la situación que se describe o constituye su explicación. El alejamiento modal no queda, no obstante, recalcado por el empleo del IND, sus formas aparecen de nuevo al lado de los marcadores temporales, remitiendo únicamente a la localización de los sucesos en el tiempo. 
enunciación (hablante - oyente) como frente al enunciado (hablante - mensaje) no puede pasar desapercibida no solamente en el caso de la selección modal tipo indicativo/subjuntivo, sino también en la elección que se está operando dentro de los pretéritos del modo indicativo. Ahora bien, el mero hecho de afirmar el predominio de la referencia temporal que se da en distintos métodos de ELE para la explicación de los tiempos del pasado, no indica en ningún modo la interpretación del dicho predominio ${ }^{3}$. Me pregunto, por consiguiente, si la temporalidad destacada en los manuales es:

1) un punto de partida establecido según la oposición sistémica de acuerdo con la tradición del análisis de tipo discreto, o/y

2) una forma de destacar la función prototípica de los tiempos verbales.

La oposición entre el PP y el IND que viene establecida en los métodos de ELE, tal vez pueda hacer pensar en la oposición no gradual de tipo binario cuyo origen se encuentra en los análisis fonológicos. Igual sería más adecuado hablar, en nuestro caso, de la diferenciación opositoria trielemental dado que además de PP e IND no se tarda en introducir el tercer tiempo del pasado, el imperfecto de indicativo, en cuya exposición, al menos en primeras aproximaciones, sobresalen referencias a las acciones que duran, que se repiten, acciones inacabadas o acciones que se desarrollan sólo una vez (puntuales) ${ }^{4}$. Así, queda subrayada la separación entre lo que se da en una presentación primaria y simplificada como puntual y terminado (IND), repetitivo e inacabado (IMP) y ocurrido [recientemente] pero no perteneciente del todo al pasado (PP). Evidentemente, tal separación no puede considerarse como válida ni siquiera aplicando criterios de índole temporal o aspectual $^{5}$, la incluyo únicamente a modo de ilustración de lo que se deduce de las oposiciones establecidas para el sistema de los pretéritos en los métodos de ELE. Así pues, para el empleo de PP e IND se crea un binarismo oposicional donde los complementos cirunstanciales de tiempo funcionan como básicos a la hora de elegir entre uno $u$ otro tiempo verbal. En este sentido, podría hablarse de una categorización discreta; los contenidos modales dejan percibirse, a lo mejor, en las contextualizaciones dialógicas, estas últimas resultan, no obstante, aisladas y raras veces explícitas. Más adecuada parecería, sin lugar a dudas, la oposición establecida de acuerdo con la teoría de la temporalidad lingüística definida por G. Rojo (1974:

${ }^{3}$ Convendría, a lo mejor, matizar dado que en uno de los métodos sometidos al análisis encontramos referencias explícitas al empleo del PP en su función emocional-actualizadora (nos referimos a Avance, Curso de español, nivel intermedio). Dicho empleo no queda, sin embargo, contextualizado en los textos o actos de habla que se proponen a continuación.

${ }^{4}$ Son, por lo tanto, de nuevo criterios extralinguísticos que parecen predominar. Desde el punto de vista modal, la idea de duración no se debe tan sólo al empleo del imperfecto, sino a una coincidencia de varios elementos, entre los que desempeñan un papel fundamental el contexto, el conocimiento que tienen el hablante y su oyente del mundo con todas las experiencias que comporta (sin mencionar ya, el semantismo de cada verbo), (F. Matte Bon, 1995: 27).

${ }^{5}$ Es una cuestión sumamente compleja, véanse a ese respecto, por ejemplo, a W. Nowikow (2002: 177-186). 
68-149) (esta última aparece como categoría deíctica mediante la cual se expresa la orientación de una situación con respecto a un punto central u origen, o bien con respecto a otro punto que, a su vez, está directa o indirectamente orientado con respecto al origen). Gracias a esta perspectiva, la temporalidad deja de coincidir con las nociones extralinguísticas de presente, pasado o futuro, y las formas temporales señalan no el tiempo de la acción, sino el orden y el aspecto de la misma en el tiempo (M. L. Gutierrez Araus, 1998: 275 ). Así, parecen menos desconcentrantes los empleos de tipo "¿Recibiste mi fax?" (la pregunta dirigida, p.ej., a un compañero de oficina) puesto que el enfoque de la temporalidad lingüística indica que la forma absoluta <hablé> compite con la forma < he hablado> considerada en sus dos referencias: como forma de anterioridad al origen y como la relacionada con un punto de simultaneidad al origen.

La separación de la perspectiva temporal, llevada a cabo incluso para cumplir el objetivo mayor, a saber, el de la claridad en la exposición didáctica, no parece del todo facilitante y debería considerarse más bien como equívoca. El influjo de los contenidos modales ha de reflejarse de cualquier modo, dado que los tiempos verbales van fuertemente marcados por la situación comunicativa en que aparecen. Lo confirman, incluso, las formas discursivas del ámbito literario: en las novelas cortas, los cuentos y las novelas dominan las formas del grupo del mundo narrado, entre ellas las formas del IND (adscritas, en otra terminología, a la perspectiva inactual), mientras que en la lítica, el drama, el ensayo biográfico, la crítica literaria y el tratado filosófico, predominan las del grupo del mundo comentado (entre ellas, PP, la forma verbal propia de la perspectiva actual) ${ }^{6}$. Como señala M. L. Gutierrez Araus (1998: 227), en la narración se da la relajación primo plano de actualidad y de dramatismo, lo que no ocurre, por supuesto, dentro del ámbito del llamado mundo comentado.

El enfoque a través del que se presenta el funcionamiento de los pretéritos españoles en los métodos de ELE parece recaicar la dimensión temporal, estableciendo una evidente jerarquía dentro de la polifuncionalidad de estos tiempos de indicativo. Si optáramos por interpretar este hecho como una puesta en relieve de la función prototípica, habría que precisar que la demarcación entre los usos prototípicos y los usos periféricos no se establece necesariamente a base de los criterios de la temporalidad lingüística (ante todo, si tomamos en cuenta la oposición entre el PP y el IND'). Así por ejemplo, vienen destacados los valores

\footnotetext{
${ }^{6}$ Recurrimos aquí a la clasificación de Weinrich (1974) quien propone dos grupos de unidades verbales, las del mundo comentado y las del mundo narrado.

${ }^{7}$ Dentro de los usos del imperfecto de indictaivo, tal y como se los presenta en los manuales de ELE sometidos al análisis, la jerarqứa aparece como más cercana al paradigma: función prototípica usos discursivos secundarios. Así, en una primera aproximación, el imperfecto se manifiesta como el tiempo que expresa la continuidad en el pasado. Las demás funciones (como, por ejemplo, las vinculadas con la cortesía o modestia verbal [imperfecto de cortesía], el discurso anterior presupuesto [imperfecto intertextual], la fantasía [imperfecto lúdico], ...) remiten a las situaciones más ocasionales y, lógicamente, no destacan en la exposición didáctica. Además, como señala J. Wilk-Racieska (2000:
} 
primarios del PP, que en el plano del sistema verbal se presenta principalmente como perfecto de anterioridad inmediata y perfecto resultativo. No se indica de forma suficiente la correlación de los factores temporales y los de carácter modal; de ahí la frecuente extrañeza por parte de los alumnos ante la posibilidad de desviar un hecho del nivel de la llamada perspectiva inactual al plano de la actualidad (y al revés).

Aventurándose en el terreno de los juegos de palabras, cabría que concluir que la modalidad implícita (dentro de la cual caben los empleos modales de los tiempos de indicativo) queda exactamente implícita, por no decir "encubierta", en la mayoría de los métodos de ELE. Los enfoques comunicativos preconizan el recurso a los contenidos funcionales y discursivos, subrayan el papel dominante de los factores comunicativos, proporcionando, sin embargo, reglas semántico-sintácticas que no siempre están en concordancia con los supuestos didácticos de base.

\section{REFERENCIAS BIBLIOGRÁFICAS}

Gutierrez Araus, M. L. (1998), Sistema y discurso en las formas verbales del pasado. Revista española de Linguística, 28, 2, 275- 300.

Matte Bon, F. (1995), Gramática Comunicativa del español. De la lengua a la idea. Madrid: Edelsa. Nowikow, W. (2002), Acerca de la distinción entre los conceptos de [Himitación temporal] y [tconclusión procesal]. Romanica Cracoviensia, 2002/2, 177-186.

Nowikow, W. (2001), La altemancia de los modos Indicativo y Subjuntivo en las cláusulas subordinadas sustantivas. Poznań: Wydawnictwo Naukowe UAM.

Rojo, G. (1974), La temporalidad verbal en español. Verba, 1, Universidad de Santiago de Compostela, 68-149.

Weinrich, H. (1968), Estructuras y función de los tiempos en el lenguaje, trad. Federico Latorre. Madrid: Gredos.

Wilk-Racięska, J. (2000), Tiempo real y tiempos gramaticales: presente e imperfecto de indicativo. Estudios de Lingüística, 14/2000, 275-290.

\section{MÉTODOS ANALIZADOS}

Artuñedo, B. y Donson, C. (1998), Ele 2. Curso de español para extranjeros. Madrid: Ediciones SM. B orobio, V. (2001), Ele l. Curso de español para extranjeros. Madrid: Ediciones SM.

Miquel, L. y Sans, N. (1989), Intercambio 1. Madrid: Difusión.

Miquel, L. y Sans, N. (1990), Intercambio 2. Madrid: Difusion.

Miquel, L. y Sans, N. (1994), Rápido. Curso intensivo de español. Barcelona: Difusión.

Moreno, C., Moreno, V., Zurita, P. (1995), Avance. Curso de español. Nivel intermedis. Alcobendas: SGEL.

Sánchez Lobato, J., Moreno García, C., Santos Gargallo, 1. (1997), Españols sin fronteras 1. Alcobendas: SGEL.

Sánchez Lobato, J., Moreno García, C., Santos Gargallo, I. (1998), Español sin fromteras 2. Alcobendas: SGEL.

284-286), esos empleos periféricos son función de la continuidad más otros factores linguísticos y extralinguísticos, siendo la continuidad el factor más importante. 